\title{
Effect of Taping and Virtual Reality Combined Exercise on Static and Dynamic Balance With Functional Ankle Instability
}

\author{
Ki-jong Kim, PT, PhD, Mi-yeong Gang, OT, PhD \\ Department of Occupational Therapy, College of Health and Welfare, Woosong University, Daejeon, Korea
}

\author{
Article Info \\ Received October 5, 2020 \\ Revised October 27, 2020 \\ Accepted October 27, 2020 \\ Corresponding Author \\ Mi-yeong Gang \\ E-mail: miyeong@wsu.ac.kr \\ https://orcid.org/0000-0002-9361-7581
}

\section{Key Words}

Ankle Joint

Athletic tape

Joint instability

Postural balance

Rehabilitation

Virtual reality

\begin{abstract}
Background: Ankle sprain is one of the most common musculoskeletal injuries in the sports population or during usual daily life activities. The sprain can cause functional ankle instability (FAI), and it is very important to treat FAl. However, the optimum intervention method for FAI has yet to be determined.
\end{abstract}

Objects: This study investigated the impact that virtual reality (VR) training program on balance with ankle kinesio taping for FAl.

Methods: Twenty-two people were selected for the study and randomly divided into the experimental $(n=11)$ and the control group $(n=11)$. The experimental group had attached kinesio taping on the ankle and then implemented a virtual reality exercise program for 30 minutes a day. Nintendo Wii Fit Plus was used for the VR intervention three times a week for four weeks. The control group performed only two measurements without intervention.

Results: There were no statistically significant differences in overall, anterior-posterior (AP), medial-lateral $(\mathrm{ML})$ index of the static balance, and significant differences in overall, $A P, M L$ index of the dynamic balance when taping and VR exercise were applied at the same time $(p<$ 0.05). There were no significant differences in overall and ML index of static and dynamic balance compared with before and after assessment between the experimental and the control group, and found differences in AP index of static and dynamic balance $(p<0.05)$.

Conclusion: Kinesio taping may not influence the balance of FAl as great as people expected. VR approach does not affect the static balance of FAI, but it influences dynamic balance in overall, AP, ML index. The authors suggest that VR-based exercises can be used as an additional concept in clinicians for FAl or as part of a home program because the exercises still have limitations.

\section{INTRODUCTION}

An ankle sprain is one of the most common musculoskeletal injuries in the sports population or during usual daily life activities [1]. Functional ankle instability (FAI) is characterized by recurrent ankle sprains and/or feelings of joint instability. The risk factors of FAI include muscle weakness and the lack of proprioceptive sense, it may lead to a significant impairment in postural control and balance [2,3].

A variety of exercise programs were used as an intervention for FAI, and conservative treatment interventions including balance, proprioceptive, and muscle strengthening exercises are effective for patients with FAI [4]. Therapeutic ankle taping is thought to enhance proprioceptive acuity by increased stim- ulation of the cutaneous mechanoreceptors [5]. In addition, taping is using to restore proper muscle function by facilitation or inhibiting muscular contraction, and to improve blood follow and lymphatic drainage [6]. However, the relationship between postural stability and ankle taping during static and dynamic balance activities is still unknown.

Virtual reality (VR) can be described as a high-quality user and computer interface that enables real-time simulation and interaction via multiple sensory systems [7]. A recently popular intervention that may improve postural control is a VR training program as physical and occupational therapy [8-11]. VR training programs were not easy to be applying due to restrictive usefulness or high cost. One of the VR devices is the Nintendo Wii Fit program is low-cost and useful for exercising, 
which consists of the exercise-based game included joint flexibility, muscular strength, and balance [12]. Despite the benefits, there is unclear the effectiveness of static and dynamic balance using the VR program and taping combined exercise on FAI. Therefore, in this study, we investigated the impact that VR training program on balance with ankle kinesio taping for FAI.

\section{MATERIALS AND METHODS}

\section{Subjects}

The subjects of this study were selected when conditions of 24 or less were met in the Cumberland ankle instability tool (CAIT) questionnaire for university students who experienced an ankle sprain in the past and felt ankle instability afterward [13]. Those with orthopedic, neurological, and other diseases that could affect the results of the study were excluded. Finally, all subjects were fully explained for the purpose and method of the study, and they voluntarily agreed to join this project. Twenty-two people (male: 5, female: 17) were selected for the study and randomly divided into the experimental $(n=11)$ and the control group $(n=11)$. The control group performed only two measurements without intervention. The general characteristics of the subjects were age $21.6 \pm 2.3$, height $166.1 \pm$ 5.9, and weight $61.7 \pm 7.8$. All the study methods and procedures were conducted in accordance with the Declaration of Helsinki.

\section{Taping Application and Virtual Reality Exercise}

The experimental group had attached kinesio taping on the ankle and then implemented the VR exercise program. The taping was wrapped twice in the figure of 8 technique from the center of the soles to the ankles for the experimental group while maintaining the dorsiflexion position (Figure 1A).

This exercise was conducted three times a week for four weeks as an ankle reinforced exercise using a VR exercise program, followed by warm-up exercise ( 5 minutes), intervention (20 minutes), and cool-down exercise (5 minutes). The research exercise carried out five strengths (e.g., lunge, single leg extension, sideways leg lift, single leg twist, rowing squat) and five balanced (e.g., soccer heading, ski slalom, tight rope walk, table tilt, snowboard slalom) exercises among the programs built into the Nintendo Wii Fit Plus (Nintendo Inc., Kyoto, Japan) for VR exercise programs (Figure 1B).
The intensity of the strength exercise in VR was set to increase the intensity of exercises by repeating it 10 times until 1 to 2 weeks, and by repeating it 20 times between 3 to 4 weeks. The balanced exercises were adjusted according to the level of development of the subjects from beginner to advance. Each VR exercise took a different time, so the authors set the total time not to exceed 20 minutes [14].

\section{Measurement}

Biodex balance system ${ }^{\circledast}$ (BBS; Biodex Medical System Inc., Shirley, NY, USA) can measure static and dynamic balance (1 to 12 levels) and can acquire data such as overall stability, anterior-posterior (AP), medial-lateral (ML) index (unit: degree). In this study, the static balance was first measured, and then the dynamic balance was measured for FAI. The static and dynamic balance used the calculated average value by measuring a total of three times each, and a single assessment was set to rest 10 seconds after evaluating 30 seconds. A total of two measurements were taken for both groups in the first week and in the fourth week, and the dynamic balance was set at level 2.

\section{Statistical Analysis}

All the data in this study were analyzed using SPSS version 25 (Statistical Package for the Social Sciences; IBM Co., Armonk, NY, USA). The data in this study were not the normal distribution. As a result, a nonparametric test was used. Mann-Whitney U-test was conducted to analyze two different groups, and the Wilcoxon signed-rank test was performed for comparison
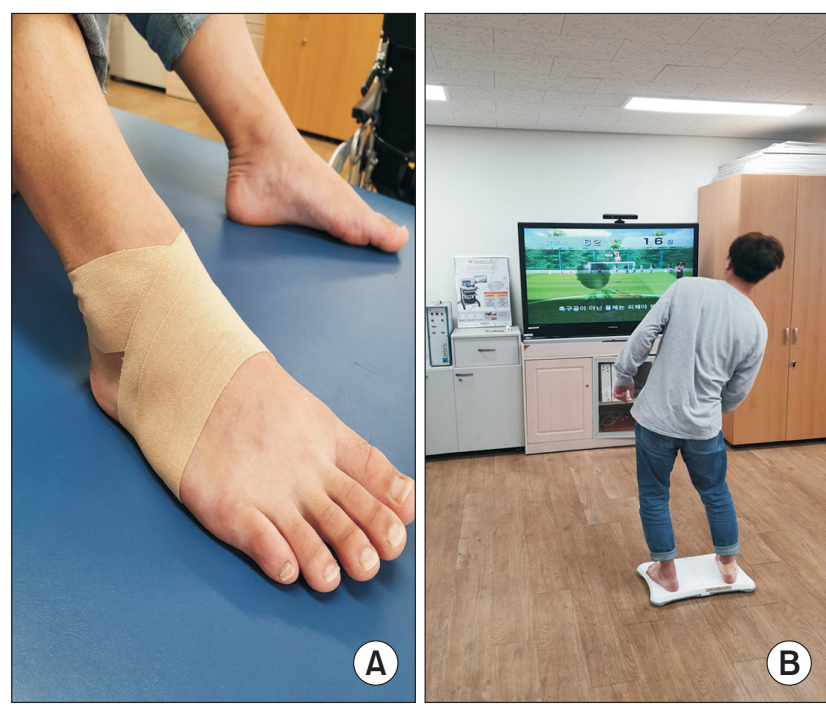

Figure 1. (A) The ankle kinesio taping and (B) the virtual reality exercise. 
before and after. The significance level was set to $\alpha=0.05$ with a two-tailed statistical test.

\section{RESULTS}

There were no statistically significant differences in overall, $\mathrm{AP}, \mathrm{ML}$ index of the static balance, and significant differences in overall, AP, ML index of the dynamic balance when taping and VR exercise were applied at the same time $(\mathrm{p}<0.05)($ Fig-

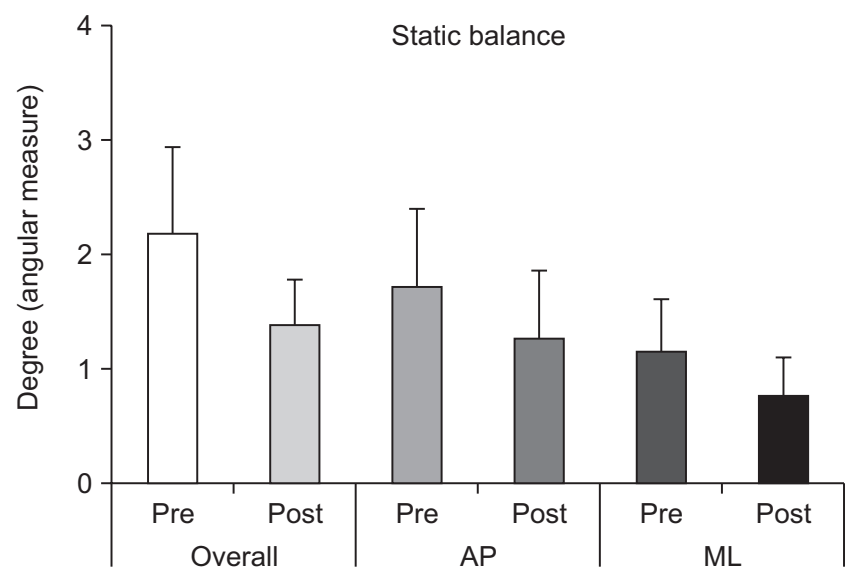

Figure 2. Comparison of static balance of the taping with the virtual reality (i.e., experimental group) before and after the intervention. AP, anterior-posterior; ML, medial-lateral. Tested by Wilcoxon signed-rank test. ures 2 and 3). There were no significant differences in overall and ML index of static and dynamic balance compared with before and after assessment between the experimental and the control group, and found differences in AP index of static and dynamic balance $(\mathrm{p}<0.05)$ (Table 1). Before and after the comparison of the control group, there were no significant differences except AP index of dynamic balance (Table 2).

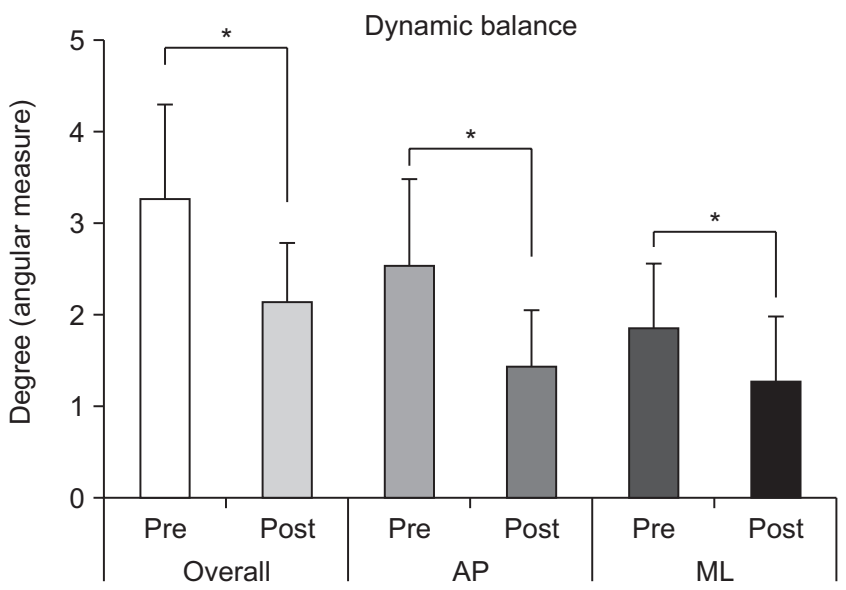

Figure 3. Comparison of dynamic balance of the taping with the virtual reality (i.e., experimental group) before and after the intervention. AP, anterior-posterior; $M L$, medial-lateral. ${ }^{*} p<0.05$, tested by Wilcoxon signedrank test.

Table 1. Comparison of the experimental and control group after the intervention

\begin{tabular}{|c|c|c|c|c|}
\hline Direction $\left({ }^{\circ}\right)$ & Experimental group & Control group & $u$ & $\mathrm{p}$-value \\
\hline \multicolumn{5}{|l|}{ Static balance } \\
\hline Overall & $1.56 \pm 0.68$ & $1.42 \pm 1.24$ & 35 & 0.090 \\
\hline$A P$ & $1.26 \pm 0.61$ & $1.09 \pm 1.12$ & 30.5 & $0.047^{*}$ \\
\hline$M L$ & $0.76 \pm 0.35$ & $0.73 \pm 0.51$ & 41 & 0.191 \\
\hline \multicolumn{5}{|l|}{ Dynamic balance } \\
\hline Overall & $2.11 \pm 0.67$ & $2.59 \pm 1.35$ & 60.5 & 1.000 \\
\hline $\mathrm{AP}$ & $1.43 \pm 0.64$ & $1.04 \pm 0.42$ & 29 & $0.038 *$ \\
\hline$M L$ & $1.26 \pm 0.73$ & $2.16 \pm 1.38$ & 36 & 0.114 \\
\hline
\end{tabular}

Values are presented as mean \pm standard deviation. AP, anterior-posterior; ML, medial-lateral. ${ }^{*} \mathrm{p}<0.05$, tested by Mann-Whitney U-test.

Table 2. Comparison of the no intervention (i.e., control) group before and after the intervention

\begin{tabular}{|c|c|c|c|c|}
\hline Direction $\left({ }^{\circ}\right)$ & Pre & Post & Z & $\mathrm{p}$-value \\
\hline \multicolumn{5}{|l|}{ Static balance } \\
\hline Overall & $1.53 \pm 1.39$ & $1.42 \pm 1.24$ & -1.070 & 0.285 \\
\hline$A P$ & $1.18 \pm 1.45$ & $1.09 \pm 1.12$ & -0.155 & 0.877 \\
\hline$M L$ & $0.68 \pm 0.24$ & $0.73 \pm 0.51$ & -0.672 & 0.502 \\
\hline \multicolumn{5}{|l|}{ Dynamic balance } \\
\hline Overall & $3.12 \pm 1.071$ & $2.59 \pm 1.35$ & -1.468 & 0.142 \\
\hline $\mathrm{AP}$ & $1.29 \pm 0.60$ & $1.04 \pm 0.42$ & -2.311 & $0.021 *$ \\
\hline$M L$ & $2.61 \pm 1.55$ & $2.16 \pm 1.38$ & -1.248 & 0.212 \\
\hline
\end{tabular}

Values are presented as mean \pm standard deviation. AP, anterior-posterior; ML, medial-lateral. ${ }^{*} \mathrm{p}<0.05$, tested by Wilcoxon signed-rank test. 


\section{DISCUSSION}

There was no significant difference in static balance, and only a significant difference found in dynamic balance when taping and virtual reality were applied. These results were different from our expectations. This is because the authors expected that a sense of balance would improve when applying combined intervention (e.g., taping and VR) or at least one intervention (e.g., taping or VR). The reasons for this are explained as follows. First, in this study, there may have been no effect on taping intervention. For instance, Raymond et al. [15] found that taping was not effective compared to the no taping condition for recurrent ankle sprain and FAI. Another study showed that the effect of taping may not be as great as expected, given that kinesio taping did not affect the reduction of acute swelling when applied to a sports athlete after an ankle sprain [16]. On the other hand, the application of VR to adults in their 20s for FAI showed significant differences in overall, AP, and ML index of the dynamic balance [17]. Of course, the authors still think that kinesio taping has positive effects, such as improving the performance of the ankle [18]. Second, Nintendo Wii Fit Plus is a tool for VR, but it was created for entertainment [19]. For example, it is difficult to set muscle strength and balanced exercise time accurately in this equipment. The reason for this is that in this study, an exercise (e.g., table tilt) can last longer depending on the achievement of the subject, and another exercise (e.g., ski slalom) must be very short. To sum up, if researchers try to apply for Nintendo equipment as a VR training, the results cannot be guaranteed. Based on the study results, the authors recommend that this equipment can use as a supporting tool for existing physical and occupational therapy not apply the main intervention of ankle rehabilitation in the clinics.

No statistically significant differences were found in the comparison of static and dynamic balances between the experimental and control groups in this study. Unexpectedly, significant decreases were seen in the control AP index of static and dynamic balance. The authors would like to explain the reasons for the unexpected results as follows. First, it is thought that the starting point of the experimental and control groups was different. In other words, because the starting point of the control was low, there seemed to be a significant difference, but it was a meaningless result. The selection criteria for this study were 24 or fewer CAIT scores. The lower the score means the more serious the FAI [20,21]. Therefore, it is shown that the large differences in individual instability of the ankle resulted in a lesser starting point for the control group than for the experimental group. A previous study also mentioned the need to sort CAIT scores by grade to select subjects for more accurate comparisons [17]. Second, this may be because, as mentioned earlier, the effect of the intervention of the experimental group was not strong. If the simultaneous application of the VR and taping were effective, different results would have been obtained. However, as mentioned earlier, taping seems ineffective, and only the VR intervention seems to have some effects on the dynamic balance of FAI.

The limitations of this study are as follows. First, only university students participated in the study. The elderly might produce different results. Second, before the trial, the CAIT score differences might exist between the experimental and control group. This is because the study defined CAIT below 24 as FAI and did not consider the difference in individual scores (range 0-24). Third, Nintendo Wii Fit Plus can be used as VR, but different results may come out if other devices or programs are applied as VR. Fourth, since the figure of 8 of kinesio taping technique was used in this study, it is hard to say that it reflects all the taping effects. In future studies, various taping techniques are necessary to apply FAI so that we can fully understand the effect. Despite having some limitations, the authors think that this article is showing meaningful results to those who work in rehabilitation areas. The reason is that we studied a new intervention approach by combining VR and taping.

\section{CONCLUSIONS}

Kinesio taping may not influence the balance of FAI as great as people expected. VR approach does not affect the static balance of FAI, but it influences dynamic balance in overall, AP, ML index. The authors suggest that VR-based exercises can be used as an additional concept in clinicians for FAI or as part of a home program because the exercises still have limitations. In addition, if Nintendo Wii Fit Plus is applied in VR with FAI, it is necessary to create a specific exercise program for clinical use.

\section{ACKNOWLEDGEMENTS}

This research is based on the support of 2020 Woosong Uni- 
versity Academic Research Funding.

\section{CONFLICTS OF INTEREST}

No potential conflict of interest relevant to this article was reported.

\section{AUTHOR CONTRIBUTIONS}

Conceptualization: KK. Data curation: KK. Formal analysis: KK. Investigation: KK. Methodology: KK. Project administration: KK. Resources: KK, MG. Software: KK. Supervision: KK. Validation: KK. Visualization: MG. Writing - original draft: KK, MG. Writing - review \& editing: KK, MG.

\section{ORCID}

Ki-jong Kim, https://orcid.org/0000-0001-7105-3415

\section{REFERENCES}

1. Doherty C, Delahunt E, Caulfield B, Hertel J, Ryan J, Bleakley C. The incidence and prevalence of ankle sprain injury: a systematic review and meta-analysis of prospective epidemiological studies. Sports Med 2014;44(1):123-40.

2. Kim KJ. Impact of combined muscle strength and proprioceptive exercises on functional ankle instability. J Int Acad Phys Ther Res 2013;4(2):600-4.

3. Obata H, Kawashima N, Ohtsuki T, Nakazawa K. Aging effects on posture-related modulation of stretch reflex excitability in the ankle muscles in humans. J Electromyogr Kinesiol 2012;22(1):31-6.

4. Loudon JK, Santos MJ, Franks L, Liu W. The effectiveness of active exercise as an intervention for functional ankle instability: a systematic review. Sports Med 2008;38(7):553-63.

5. Feuerbach JW, Grabiner MD, Koh TJ, Weiker GG. Effect of an ankle orthosis and ankle ligament anesthesia on ankle joint proprioception. Am J Sports Med 1994;22(2):223-9.

6. Halseth T, McChesney JW, Debeliso M, Vaughn R, Lien J. The effects of kinesio ${ }^{\mathrm{TM}}$ taping on proprioception at the ankle. J Sports Sci Med 2004;3(1):1-7.

7. Burdea GC. Virtual rehabilitation--benefits and challenges. Methods Inf Med 2003;42(5):519-23.

8. Cho KH, Lee KJ, Song CH. Virtual-reality balance training with a video-game system improves dynamic balance in chronic stroke patients. Tohoku J Exp Med 2012;228(1):69-74.

9. Kim KJ, Heo M. Effects of virtual reality programs on balance in functional ankle instability. J Phys Ther Sci 2015;27(10):3097-101.

10. Saldana D, Neureither M, Schmiesing A, Jahng E, Kysh L, Roll SC, et al. Applications of head-mounted displays for virtual reality in adult physical rehabilitation: a scoping review. Am J Occup Ther 2020;74(5):7405205060p1-15.

11. Weber H, Barr C, Gough C, van den Berg M. How commercially available virtual reality-based interventions are delivered and reported in gait, posture, and balance rehabilitation: a systematic review. Phys Ther 2020;100(10):1805-15.

12. Taylor MJ, McCormick D, Shawis T, Impson R, Griffin M. Activity-promoting gaming systems in exercise and rehabilitation. J Rehabil Res Dev 2011;48(10):1171-86.

13. Kim KJ, Jegal H, Jun HJ, Choi BJ, Choi HJ, Yu SH, et al. The comparison of balance using Cumberland ankle instability tool to stable and instability ankle. J Korean Soc Phys Med 2013;8(3):361-8.

14. Kim KJ, Heo M. Comparison of virtual reality exercise versus conventional exercise on balance in patients with functional ankle instability: a randomized controlled trial. J Back Musculoskelet Rehabil 2019;32(6):905-11.

15. Raymond J, Nicholson LL, Hiller CE, Refshauge KM. The effect of ankle taping or bracing on proprioception in functional ankle instability: a systematic review and meta-analysis. J Sci Med Sport 2012;15(5):386-92.

16. Nunes GS, Vargas VZ, Wageck B, Hauphental DP, da Luz CM, de Noronha M. Kinesio taping does not decrease swelling in acute, lateral ankle sprain of athletes: a randomised trial. J Physiother 2015;61(1):28-33.

17. Kim KJ, Yoo CU. Comparison of effects after applying virtual reality ankle exercise and high-frequency current to functional ankle instability. Korean J Neuromuscul Rehabil 2020;10(2):87-93.

18. Wang Y, Gu Y, Chen J, Luo W, He W, Han Z, et al. Kinesio taping is superior to other taping methods in ankle functional performance improvement: a systematic review and metaanalysis. Clin Rehabil 2018;32(11):1472-81.

19. Merchant-Borna K, Jones CM, Janigro M, Wasserman EB, Clark RA, Bazarian JJ. Evaluation of Nintendo Wii balance board as a tool for measuring postural stability after sport-related concussion. J Athl Train 2017;52(3):245-55. 
20. Hiller CE, Refshauge KM, Bundy AC, Herbert RD, Kilbreath SL. The Cumberland ankle instability tool: a report of validity and reliability testing. Arch Phys Med Rehabil 2006;87(9):123541.
21. Rosen A, Ko J, Brown C. A multivariate assessment of clinical contributions to the severity of perceived dysfunction measured by the Cumberland ankle instability tool. Int J Sports Med 2016;37(14):1154-8. 